\title{
Effects of mutant TDP-43 on the Nrf2/ARE pathway and protein expression of MafK and JDP2 in NSC-34 cells
}

\author{
Y.P. Tian', F.Y. Che ${ }^{1,2}$, Q.P. Su ${ }^{2}$, Y.C. Lu ${ }^{2}$, C.P. You ${ }^{2}$, L.M. Huang ${ }^{3}$, \\ S.G. Wang ${ }^{4}$, L. Wang ${ }^{2}$ and J.X. Yu ${ }^{1,2}$ \\ ${ }^{1}$ Department of Neurology, Linyi People's Hospital, Linyi, Shandong, China \\ ${ }^{2}$ Central Laboratory, Linyi People's Hospital, Linyi, Shandong, China \\ ${ }^{3}$ Department of Emergency, Linyi People's Hospital, Linyi, Shandong, China \\ ${ }^{4}$ Department of Neurology, Rizhao People's Hospital, Rizhao, Shandong, China \\ Corresponding author: J.X. Yu \\ E-mail: yujixu@yahoo.com \\ Genet. Mol. Res. 16 (2): gmr16029638 \\ Received February 1, 2017 \\ Accepted March 31, 2017 \\ Published May 10, 2017 \\ DOI http://dx.doi.org/10.4238/gmr16029638
}

Copyright $(2017$ The Authors. This is an open-access article distributed under the terms of the Creative Commons Attribution ShareAlike (CC BY-SA) 4.0 License.

\begin{abstract}
Amyotrophic lateral sclerosis (ALS) is a neurodegenerative disease that affects motor neurons and lacks an effective treatment. The disease pathogenesis has not been clarified at present. Pathological transactive response DNA-binding protein 43 (TDP-43) plays an important role in the pathogenesis of ALS. Nuclear translocation of nuclear factor erythroid 2 (NF-E2)-related factor 2 (Nrf2) is found in a mutant TDP-43 transgenic cell model, but its downstream antioxidant enzyme expression is decreased. To elucidate the specific mechanism of Nrf2/ARE (antioxidant responsive element) signaling dysfunction, we constructed an ALS cell model with human mutant TDP-43 using the NSC-34 cell line to evaluate the impact of the TDP-43 mutation on the Nrf2/ARE pathway. We found the nuclear translocation of Nrf2, but the expression of total Nrf2, cytoplasmic Nrf2, and downstream phase II detoxifying enzyme (NQO1) was decreased in NSC-34 cells transfected with the TDP-43-M337V plasmid. Besides,
\end{abstract}


TDP-43-M337V plasmid-transfected NSC-34 cells were rounded with reduced neurites, shortened axons, increased levels of intracellular lipid peroxidation products, and decreased viability, which suggests that the TDP-43-M337V plasmid weakened the antioxidant capacity of NSC-34 cells and increased their susceptibility to oxidative damage. We further showed that expression of the MafK protein and the Jun dimerization protein 2 (JDP2) was reduced in TDP-43-M337V plasmid-transfected NSC-34 cells, which might cause accumulation of Nrf2 in nuclei but a decrease in NQO1 expression. Taken together, our results confirmed that TDP-43-M337V impaired the Nrf2/ARE pathway by reducing the expression of MafK and JDP2 proteins, and provided information for further research on the molecular mechanisms of TDP-43-M337V in ALS.

Key words: Amyotrophic lateral sclerosis; TDP-43; Nrf2; MafK; JDP2

\section{INTRODUCTION}

Amyotrophic lateral sclerosis (ALS) is a motor neuron disorder that manifests as a chronic, progressive, and fatal neurodegenerative disease (Zarei et al., 2015). At present, the disease pathogenesis has not been clarified, and no effective treatment is available. ALS patients typically die from respiratory muscle paralysis within 3 to 5 years after diagnosis (Mathis et al., 2017). Current evidence suggests that degeneration and death of motor neurons in ALS models are caused by a series of interacting mechanisms including oxidative stress, excitotoxicity, mitochondrial dysfunction, abnormal protein aggregation, cytoskeletal abnormalities, and genetic factors (Wang et al., 2014; Tafuri et al., 2015; Bozzo et al., 2017; Taylor et al., 2016). Of these mechanisms, oxidative stress is considered the major factor in degeneration of motor neurons.

Abnormally ubiquitinated protein aggregates, of which the main component is the transactive response DNA-binding protein 43 (TDP-43) (Neumann et al., 2006), were found in both nuclei and cytoplasm of neurons and glia in ALS patients and patients with frontotemporal dementia. Normal TDP-43 is a protein encoded by the TARDBP gene and consists of 414 amino acids with a relative molecular mass of $43 \mathrm{kDa}$ (Romano et al., 2015). It contains 2 RNA recognition sites and a glycine end that binds to single-stranded DNA and regulates transcription and splicing (Ayala et al., 2008). Physiological TDP-43 proteins are primarily located in nuclei and rarely appear in the cytoplasm (Scotter et al., 2015). TDP-43 protein expression is regulated by many signals such as those for nuclear localization and metastasis. Also, TARDBP gene mutation, protein phosphorylation, $\mathrm{N}$-terminal truncation, and ubiquitination may cause the production of pathological TDP-43 protein (Winton et al., 2008). The pathological TDP-43 protein appears as nuclear inclusion bodies, cytoplasmic aggregates, and other pathomorphological changes under disease and stress conditions (Tan et al., 2007; Maekawa et al., 2009; Tateishi et al., 2010). Previous studies have shown that TDP-43 proteins in central nervous system neurons of patients who died of ALS shift from nuclei to the cytoplasm and diffuse in ubiquitin-positive inclusion bodies (Lagier-Tourenne and Cleveland, 2009; Brown et al., 2012). Pathological TDP-43 plays a key role in ALS pathogenesis, but the specific mechanism remains unclear.

Genetics and Molecular Research 16 (2): gmr16029638 
Studies have shown nuclear translocation of the nuclear factor erythroid 2 (NF-E2)related factor 2 (Nrf2) in a mutant TDP-43 transgenic cell model, but the expression of its downstream antioxidant enzyme is decreased (Duan et al., 2010; Wang et al., 2014). Thus, when cells are under oxidative stress, Nrf2 translocates from the cytoplasm to nuclei, and expression of the downstream antioxidant enzyme decreases. This response may be associated with the dysfunction of the Nrf2/antioxidant responsive element (ARE) signaling pathway.

To elucidate the specific mechanism of Nrf2/ARE signaling dysfunction, we constructed an in vitro model of ALS with human mutant TDP-43 using a neuroblastoma spinal cord (NSC-34) hybrid cell line to evaluate the impact of the TDP-43 mutation on the Nrf2/ ARE pathway and related protein expression. Results of this study may provide experimental and theoretical evidence for ALS pathogenesis and treatment.

\section{MATERIAL AND METHODS}

\section{Materials}

The pDEST30-EGFP, pDEST30-EGFP-TDP-43-WT, and pDEST30-EGFP-TDP-43M337V plasmids were gifts from King's College London (London, UK). Lipofectamine ${ }^{\mathrm{TM}} 2000$ was purchased from Invitrogen (La Jolla, CA, USA). G418 was purchased from Sigma (St. Louis, MO, USA). Primary antibodies for MafK, Jun dimerization protein 2 (JDP2), NADPH quinone dehydrogenase 1 (NQO1), Nrf2, and $\beta$-actin, as well as secondary antibodies, were purchased from Santa Cruz Biotechnology (Santa Cruz, CA, USA). Primary antibody for SMI32 was purchased from Covance Inc. (Princeton, NJ, USA). The bicinchoninic acid protein assay kits were purchased from Tiangen Biotech Co., Ltd. (Beijing, China). Total protein and cytoplasmic and nuclear protein extraction kits were purchased from Keygen Biotech Co., Ltd. (Nanjing, China). The immunohistochemistry kits were purchased from Beijing Zhong Shan-Golden Bridge Biotechnology Co., Ltd. (Beijing, China). MDA detection kits and MTT cell proliferation and cytotoxicity assay kits were purchased from Jiancheng Bioengineering Institute (Nanjing, China).

\section{Cell culture and plasmid transfection}

NSC-34 cells were cultured in DMEM containing 10\% fetal bovine serum with penicillin-streptomycin (100 IU/mL penicillin and $0.1 \mathrm{mg} / \mathrm{mL}$ streptomycin) and incubated at $37^{\circ} \mathrm{C}$ in an incubator containing $5 \% \mathrm{CO}_{2}$. The culture medium was changed every 2-3 days until the cells grew to $90 \%$ confluence for later experiments. Cultured cells were washed twice with PBS, followed by trypsinization under observation with an inverted microscope (Ti-S, Nikon, Japan). Once retraction of processes was observed, serum containing DMEM was immediately added to stop trypsinization. The medium was then pipetted up and down to loosen cells adhered to the culture dish wall and to prepare the cell suspension. Next, 1 x $10^{4}$ cells were added to each well on a 24-well plate, followed by addition of $100 \mu \mathrm{L}$ medium per well.

The pDEST30-EGFP, pDEST30-EGFP-TDP-43-WT, and pDEST30-EGFP-TDP-43M337V plasmids were purified according to the manufacturer's instructions for the TIANprep Mini Plasmid Kit (Tiangen, Beijing, China). The plasmids were individually transfected into NSC-34 cells using Lipofectamine ${ }^{\mathrm{TM}} 2000$ reagent (Invitrogen) according to the manufacturer's instructions. Once the transfected cells reached $\sim 90 \%$ confluence, G418 working solution

Genetics and Molecular Research 16 (2): gmr16029638 
(final concentration $400 \mu \mathrm{g} / \mathrm{mL}$ ) was added, and complete culture medium containing 400 $\mu \mathrm{g} / \mathrm{mL}$ G418 was changed every other day. Non-transfected NSC-34 cells were used as negative controls. After culturing the cells for $\sim 7$ days, many dead cells were observed. The concentration of G418 in the complete culture medium was then decreased to $200 \mu \mathrm{g} / \mathrm{mL}$ for another 7 days. After culturing the cells for $\sim 14$ days, all plasmid-transfected groups had some cells survived, while all cells in the negative control group died. The transfected cells were subsequently incubated with standard medium to expand the culture, followed by repeating the above steps for a second G418 screening. After the second screening, cell purity of the transfected plasmid group increased. After expanding the culture for 5 days, the cells were observed under an inverted fluorescence microscope (Ti-S). Cells with green fluorescence were considered positive. NSC-34 cells with stably transfected pDEST30-EGFP, pDEST30EGFP-TDP-43-WT, and pDEST30-EGFP-TDP-43-M337V were designated the EGFP, TDP43-WT, and TDP-43-M337V groups, respectively.

\section{Identification of transfected cells}

\section{Observation of pDEST30-EGFP green fluorescence expression}

Green fluorescence expression of cells in culture flasks of the EGFP, TDP-43-WT, and TDP-43-M337V groups were observed under an inverted fluorescence microscope.

\section{Identification of stably expressing cell lines using RT-PCR}

Total RNA was isolated from harvested cells of the EGFP, TPD-43-WT, and TDP43-M337V groups, followed by synthesis of the first-strand cDNA using a first-strand cDNA synthesis kit (Fermentas, Vilnius, Lithuania). One microliter of the reverse transcription reaction mix was used as a template for PCR. Reaction conditions for PCR were as follows: $95^{\circ} \mathrm{C}$ denaturation for $5 \mathrm{~min} ; 30$ cycles of $94^{\circ} \mathrm{C}$ denaturation for $30 \mathrm{~s}, 59^{\circ} \mathrm{C}$ annealing for 30 $\mathrm{s}$, and $72^{\circ} \mathrm{C}$ elongation for $30 \mathrm{~s} ; 72^{\circ} \mathrm{C}$ elongation for $10 \mathrm{~min}$ and $4^{\circ} \mathrm{C}$ to terminate the reaction. PCR amplification products were separated by electrophoresis on a $2 \%$ agarose gel, followed by analysis using a gel imaging system (Bio-Rad, USA). Specific PCR primers were designed based on human TDP-43, mouse TDP-43, and $\beta$-actin sequences listed in GenBank using the Primer Premier 5.0 software (Premier Co., Canada) (Table 1).

Table 1. Sequences of RT-PCR primers.

\begin{tabular}{l|l}
\hline Primer name & Sequence \\
\hline Human TDP-43 & Forward: 5'-TCATCCCCAAGCCATTCA-3' \\
\hline Mouse TDP-43 & Reverse: 5'-CGCACCAAAGTTCATCCC-3' \\
\hline \multicolumn{1}{l}{-actin } & Forward: 5'-CTAAGCAAAGCCCAGACGAG-3' \\
\hline & Reverse: 5'- CTGATTCCCAAAGCCACCT-3' \\
\hline & Forward: 5'-GGGACCTGACTGACTACCTCA-3' \\
\hline
\end{tabular}

\section{Western blot analysis for identification of stably expressing cell lines}

Appropriate amounts of total protein from harvested cells of the EGFP, TDP-43-WT, and TDP-43-M337V groups were mixed with loading buffer, and proteins were denatured

Genetics and Molecular Research 16 (2): gmr16029638 
in a boiling water bath. An SDS-PAGE gel was used to separate total proteins, followed by transferring to a PVDF membrane and blocking of non-specific antigens. Anti-TDP-43 primary antibodies (mouse anti-human TDP-43 and rabbit anti-mouse TDP-43) were incubated separately with each protein blot at $4{ }^{\circ} \mathrm{C}$ overnight; then, incubating with the appropriate secondary antibody at room temperature for $1 \mathrm{~h}$. Immunodetection was performed with an enhanced chemiluminescent substrate.

\section{Immunocytochemistry}

After trypsinization, centrifugation, and resuspension, $2 \times 10^{4}$ cells were added to each well on a 6-well plate and incubated for $24 \mathrm{~h}$. Cells were then fixed in $4 \%$ formaldehyde for $40 \mathrm{~min}$, permeabilized with $1 \%$ Triton X-100 solution for $20 \mathrm{~min}$, and quenched in $3 \% \mathrm{H}_{2} \mathrm{O}_{2}$ for $10 \mathrm{~min}$. Non-specific antigens were blocked for $15 \mathrm{~min}$, and cells were then incubated with anti-SMI-32 primary antibody at $4^{\circ} \mathrm{C}$ overnight. Next, cells were incubated with biotin-labeled goat anti-mouse $\operatorname{IgG}$ at $37^{\circ} \mathrm{C}$ for $15 \mathrm{~min}$, followed by incubation with streptavidin-biotin HRP working solution at $37^{\circ} \mathrm{C}$ for $15 \mathrm{~min}$ and color development using 3,3'-diaminobenzidine substrate solution. After dehydration, xylene clearing, and gelatin and resin mounting of the cells on glass slides, changes in cytomorphology were observed under a microscope (CX31RTSF, Olympus, Japan).

\section{Measurement of intracellular lipid peroxidation levels}

Levels of lipid peroxidation in cells were measured by quantifying the content of the lipid peroxidation product, malondialdehyde (MDA). Among the lipid peroxidation products, MDA reacts with thiobarbituric acid to form a red product that has a maximum absorption peak at $532 \mathrm{~nm}$. According to the manufacturer's instructions of the MDA detection kit, absorbance at $523 \mathrm{~nm}$ was measured, and MDA content was calculated as nmol/mg protein.

\section{Cell viability detected by MTT assay}

MTT can be added to the cell culture medium. It is reduced by mitochondrial succinate dehydrogenase of living cells to form an insoluble dark purple crystal product, formazan; however, MTT cannot be reduced in dead cells. Formazan completely dissolves in DMSO, and its absorbance in the near wavelength of $570 \mathrm{~nm}$ can be measured by a microplate reader. We measured MTT absorbance following the manufacturer's instructions for the MTT assay.

\section{Western blots}

Total cell protein was extracted according to the manufacturer's instructions for the protein extraction kit, followed by quantifying protein concentration. Appropriate amounts of total protein were added to loading buffer and denatured in a boiling water bath, followed by SDS-PAGE separation and transfer to a PVDF membrane. The protein blot was blocked for 1 $\mathrm{h}$ and incubated with primary antibody at $4^{\circ} \mathrm{C}$ overnight. After washing with TBST, the protein blot was incubated with the relevant secondary antibody at room temperature for $1 \mathrm{~h}$, followed by washing with TBST. Each protein blot was developed using an ECL solution, followed by film exposure, developing, fixing, and imaging. After scanning the protein blot using an

Genetics and Molecular Research 16 (2): gmr16029638 
image, grayscales of the target protein and $\beta$-actin bands in the same lane were measured to calculate the ratio. Experiments were repeated independently at least three times.

\section{Statistical analysis}

Data were analyzed using analysis of variance and are reported as means \pm standard deviation. $\mathrm{P}<0.05$ was considered statistically significant. The statistical analysis was performed using the SAS System for Windows V8 (SAS Institute Inc., Cary, NC, USA).

\section{RESULTS}

\section{Observation of green fluorescence}

The 3 groups of cells were subcultured until reaching $90 \%$ confluence, followed by observation of green fluorescence under an inverted fluorescence microscope. Green fluorescence was detected in the transfected cells, which was associated with expression of transfected TDP-43 (Figure 1A, B, and C).

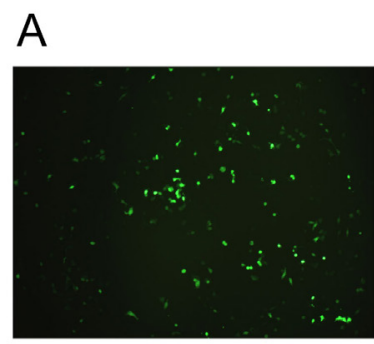

D

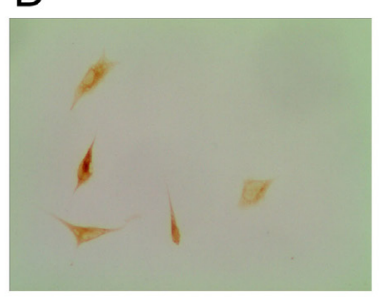

EGFP

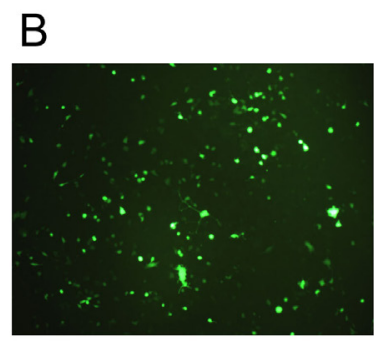

$E$

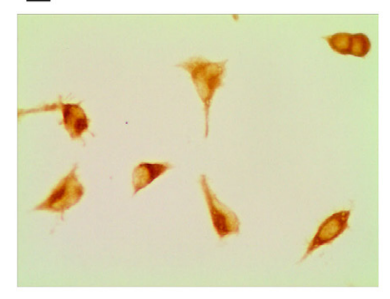

TDP-43-WT

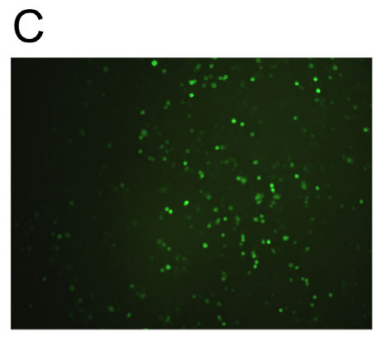

$\mathrm{F}$

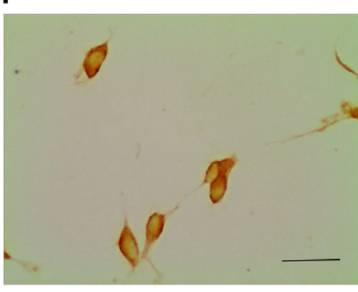

TDP-43-M337V

Figure 1. A. B. C. Representative images showing green fluorescent expression in the EGFP, TDP-43-WT, and TDP43-M337V groups (40X magnification). D. E. F. Representative images of immunocytochemical results of the EGFP, TDP-43-WT, and TDP-43-M337V groups observed by light microscopy (200X magnification, scale bar $=100 \mu \mathrm{m})$.

\section{Cytomorphological changes}

Immunocytochemistry was used to monitor morphological changes of the cells. Compared with EGFP group cells, cells of the TDP-43-M337V group had rounded cell bodies, reduced numbers of processes, and shorter axons (Figure 1D, E, and F). 


\section{TDP-43 expression in transfected cells}

RT-PCR was used to detect expression of endogenous TDP-43 (originating from NSC-34 cells) and exogenous TDP-43 (human TDP-43, from the transfected plasmid). No exogenous expression of TDP-43 was detected in cells of the EGFP group, whereas cells of both the TDP-43-M337V and TDP-43-WT groups had an exogenous expression of TDP-43. Endogenous expression of TDP-43 was detected in all 3 groups of cells (Figure 2A and B).

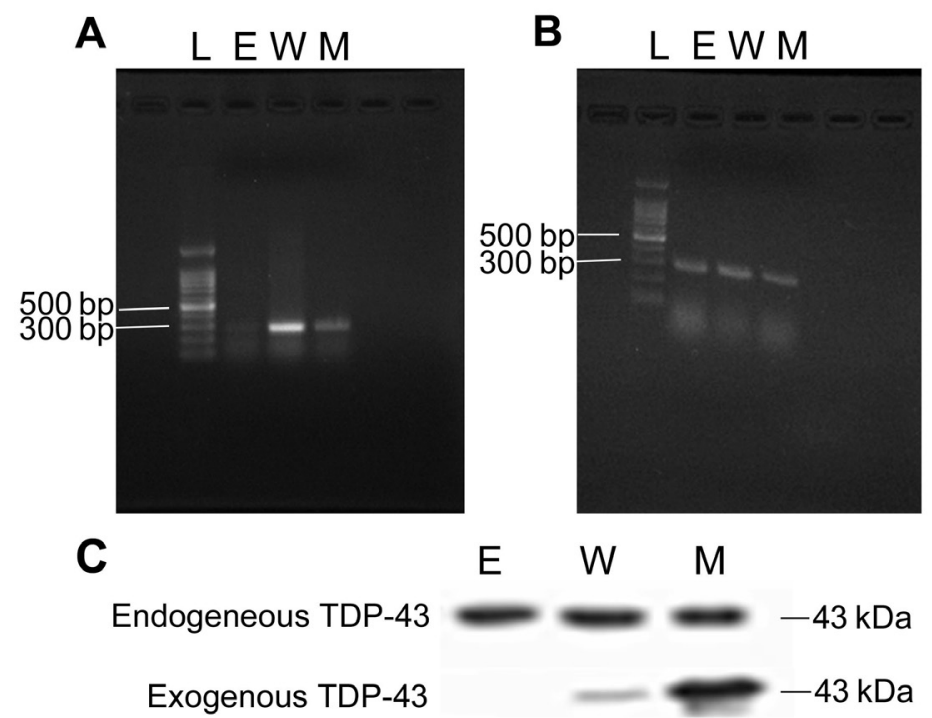

Figure 2. Endogenous and exogenous TDP-43 expression measured by RT-PCR and western blot in the 3 groups of cells. A. 284-bp exogenous TDP-43 products were detected in the TDP-43-WT and TDP-43-M337V groups. B. 320bp endogenous TDP-43 products were detected in all 3 groups. C. The endogenous TDP-43 protein was expressed in all 3 groups of cells, while the exogenous TDP-43 protein was only expressed in the TDP-43-WT and TDP-43-M337V groups. Lane L: DNA ladder, lane E: EGFP group, lane W: TDP-43-WT group, lane M: TDP-43-M337V group.

\section{TDP-43 protein expression in transfected cells}

Western blots were used to assess protein expression of endogenous and exogenous TDP-43 in the cells. The results showed that there was no detectable exogenous TDP-43 protein expression in the EGFP group, whereas cells of both the TDP-43-WT and TDP-43M337V groups had exogenous TDP-43 protein expression. The endogenous TDP-43 protein was detected in all 3 groups of cells (Figure 2C).

\section{Detection of MDA}

MDA is the final product of lipid peroxidation. Measurement of MDA levels directly reflects levels of intracellular lipid peroxidation. Once cells reached $\sim 90 \%$ confluence, the 3 groups of cells were collected to measure MDA levels. Our results showed that MDA content of the TDP-43-M337V group was significantly higher than that of the other 2 groups of cells 
$(\mathrm{P}<0.01)$, while no significant difference in MDA content was found between cells of the EGFP and TDP-43-WT groups (Figure 3A).

A

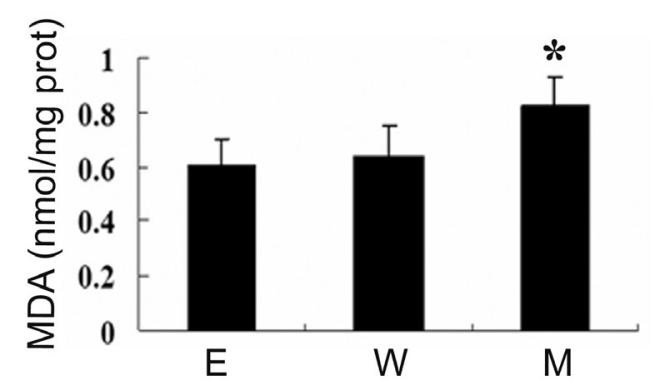

B

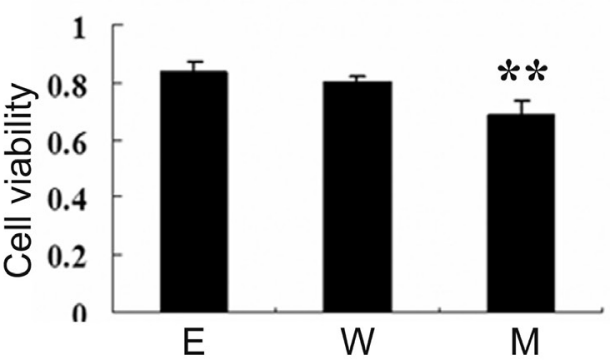

Figure 3. A. Histogram showing contents of the lipid peroxidation product, MDA, in the 3 groups. MDA content of the TDP-43-M337V group was significantly higher than that of the other 2 groups of cells $(\mathrm{N}=5, * \mathrm{P}<0.01)$. B. MTT assay of the 3 groups of cells. The viability of cells in the TDP-43-M337V group was significantly lower than that of the other 2 groups of cells $(\mathrm{N}=4, * * \mathrm{P}<0.05)$. Column E: EGFP group, column W: TDP-43-WT group, column M: TDP-43-M337V group.

\section{Measurement of cell viability using the MTT assay}

Detection of cell viability using the MTT assay showed that the MTT value of cells in the TDP-43-M337V group was significantly lower than that of the other 2 groups of cells (P $<0.05$ ), while no significant difference in MTT values was found between cells of the EGFP and TDP-43-WT groups ( $P>0.05$, Figure 3B). These results suggest that cell viability of the TDP-43-M337V group was lower than that of the other 2 groups of cells.

\section{Impact of PDEST30-EGFP-TDP-43-M337V plasmid transfection on protein expression of Nrf2 and of phase II detoxifying enzyme in NSC-34 cells}

The present experiments indicated that levels of intracellular oxidative damage after transfection with pDEST30-EGFP-TDP-43-M337V were significantly enhanced. Thus, we extracted total protein, nuclear protein, and cytoplasmic protein from the 3 groups of cells to evaluate the impact of pDEST30-EGFP-TDP-43-M337V transfection on the Nrf2-ARE antioxidant pathway. The results showed that expression of the total Nrf2 protein (Figure 4A and D) and the cytoplasmic Nrf2 protein (Figure 4B and E) was reduced, and expression of the nuclear Nrf2 protein (Figure 4B and F) was increased in cells of the TDP-43-M337V group. Besides, expression of the downstream effector, NQO1, was reduced (Figure 4C and G), which suggests that pDEST30-EGFP-TDP-43-M337V impaired the Nrf2-ARE pathway in NSC-34 cells.

\section{MafK and JDP2 protein expression}

To investigate further the impact of transfected pDEST30-EGFP-TDP-43-M337V on the intracellular Nrf2/ARE pathway, we evaluated the expression of MafK and JDP2 proteins 
in the 3 groups of cells. Our results showed that protein expression of MafK and JDP2 in cells of the TDP-43-M337V group was significantly reduced (Figure 5, P $<0.05$ ).
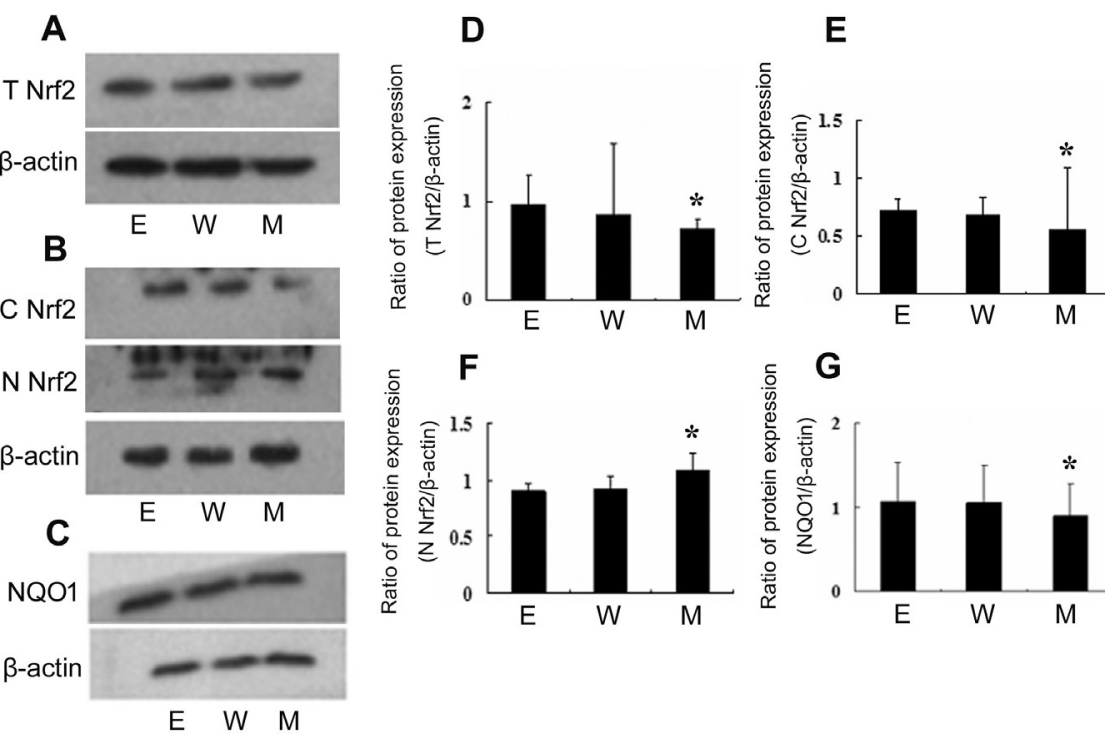

Figure 4. Nrf2 and NQO1 protein expression in the 3 groups of cells. A. B. D. E. F. Expressions of total Nrf2, cytoplasmic Nrf2, and nuclear Nrf2 protein of the TDP-43-M337V group were significantly different from those in the EGFP and the TDP-43-WT groups $(\mathrm{N}=3, * \mathrm{P}<0.05)$. C. G. NQO1 protein expression of the TDP-43-M337V group was significantly different from that in the other 2 groups of cells $\left(\mathrm{N}=3\right.$, $\left.{ }^{*} \mathrm{P}<0.05\right)$. $\mathrm{T}$ Nrf2: Total Nrf2; $\mathrm{C}$ Nrf2: cytoplasmic Nrf2; N Nrf2: nuclear Nrf2; E: EGFP group; W: TDP-43-WT group; M: TDP-43-M337V group.
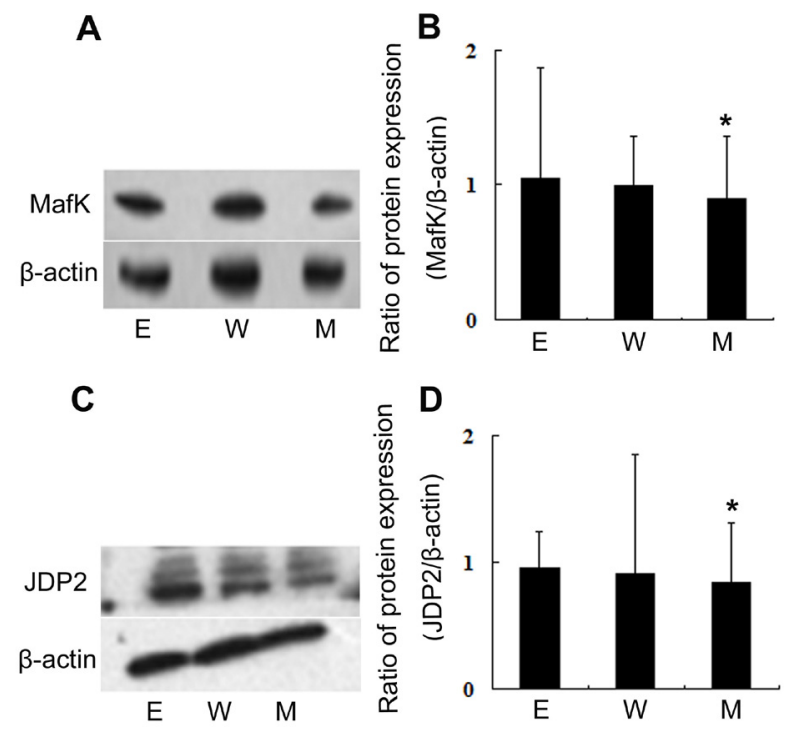

Figure 5. MafK and JDP2 protein expression in the 3 groups of cells. MafK (A and B) and JDP2 (C and D) protein expression in the TDP-43-M337V group was significantly different from that in the other 2 groups of cells $(\mathrm{N}=3$, $* \mathrm{P}<0.05)$. E: EGFP group; W: TDP-43-WT group; M: TDP-43-M337V group. 


\section{DISCUSSION}

Nrf2 regulation of an antioxidant enzyme through interaction with ARE is considered an important endogenous antioxidant pathway (Buendia et al., 2016). Under normal conditions, Nrf2 in the cytoplasm is bound to its inhibitory protein, Keapl, and remains in an inactive state. When cells are under oxidative stress, oxygen free radicals dissociate Nrf2 and Keapl to promote translocation of Nrf2 from the cytoplasm to nuclei ( $\mathrm{Li}$ and Kong, 2009). Inside the nucleus, Nrf2 binds to Maf, ATF4, cJunD, JunD, and other trans-acting elements to form heterodimers and subsequently binds to the promoter region of the corresponding cis-acting element, ARE (Kimura et al., 2007). This, in turn, activates downstream target genes of Nrf2, such as glutamylcysteine synthetase and glutathione S-transferase, and catalase expression to upregulate expression of phase II detoxifying enzymes and antioxidant proteins (Wakabayashi et al., 2004; Yamazaki et al., 2015).

Many studies have demonstrated changes in the Nrf2/ARE signaling pathway in ALS, with decreases in cellular levels of total and cytoplasmic Nrf2 protein and increases in nuclear Nrf2 protein. However, expression of downstream phase II enzymes is reduced (Duan et al., 2010; Wang et al., 2014). In this study, levels of total and cytoplasmic Nrf2 protein were reduced, and nuclear Nrf2 accumulated in NSC-34 cells after transfection with pDEST30EGFP-TDP-43-M337V. Also, the expression of the phase II detoxifying enzyme (NQO1) was reduced in NSC-34 cells transfected with pDEST30-EGFP-TDP-43-M337V. Compared with cells in the EGFP group, NSC-34 cells transfected with pDEST30-EGFP-TDP-43-M337V had rounded cell bodies, a reduced number of processes, and shorter axons. Moreover, intracellular MDA content in the TDP-43-M337V group was increased, and viability of the cells was decreased, which suggests that transfection with pDEST30-EGFP-TDP-43-M337V weakened the antioxidant capacity of NSC-34 cells and made them more susceptible to oxidative stress.

To elucidate accumulation of Nrf2 in the nuclei of NSC-34 cells and clarify the cause of reduction of the downstream phase II detoxifying enzyme after transfection with TDP-43M337V, we studied changes of the Maf family protein and JDP2 in the Nrf2/ARE pathway. Maf family proteins are important transcription factors in nuclei that mainly play roles in protein expression, cell differentiation, and apoptosis (Katsuoka and Yamamoto, 2016). Common motifs of Maf family proteins include a basic leucine zipper (b-Zip) sequence and a conserved alkaline domain, which mediate protein dimer formation and DNA binding, respectively (Katsuoka and Yamamoto, 2016). Small Maf protein is a member of the Maf protein family that has no transcriptional domain but plays a positive transcriptional regulatory role via binding to other transcriptionally active b-Zip proteins such as the activator protein 1 (AP-1) family (Kataoka et al., 1994). The large subunit p45, Nrf1, and Nrf2 are intracellular transcriptional activators of AP-1 (c-Fos, c-Jun), CREB, CNC protein, and NF-E2 (Kataoka et al., 1994). Small Maf proteins can be used as chaperones of these transcription factors involved in the formation of heterodimers and subsequently bind to the corresponding cis-acting element in DNA to promote gene transcription. They can also be used as transcription inhibitors through their formation of homodimers to inhibit gene transcription (Dhakshinamoorthy and Jaiswal, 2000). Studies have shown that Nrf2 and small Maf proteins form heterodimers to promote Nrf2 target gene expression (Katsuoka et al., 2005; Kimura et al., 2007). In this study, expression of the small Maf protein, MafK, was reduced in NSC-34 cells transfected with the TDP-43-M337V plasmid, thereby affecting the expression of Nrf2 and its downstream targeted genes. In vivo functions of Maf family proteins are complex. Small Maf proteins synergize or inhibit other transcription factors to play regulatory roles.

Genetics and Molecular Research 16 (2): gmr16029638 
JDP2 is a protein isolated from the c-Jun binding protein and consists of 163 amino acids with a b-Zip domain (Tsai et al., 2016). JDP2, as an AP-1 inhibitory protein, has been gradually recognized. It combines with other JDP2s, c-Jun, JunB, JunD, or ATF-2 to form dimers and inhibit transcriptional activity of Jun, c-Fos, and ATF-2 (Aronheim et al., 1997; Jin et al., 2001; Weidenfeld-Baranboim et al., 2009). Besides, JDP2 not only regulates gene transcription by changing chromatin structure but also recruits recombinant histone deacetylase or directly binds to histones to inhibit histone acetylation and regulate DNA, chromatin, protein, and other levels of gene transcription. Hence, JDP2 plays important roles in a variety of physiological and pathological processes in cells (Aronheim et al., 1997; Katz et al., 2001; Jin et al., 2002, 2006; Rasmussen et al., 2009). Studies have shown that JDP2 acts as a cofactor in gene transcription and plays an important role in the Nrf2/ARE/MafKmediated anti-oxidative stress transcriptional pathway (Chiou et al., 2013; Tanigawa et al., 2013). JDP2 directly binds to the core sequence of ARE and Nrf2-MafK through the basic leucine zipper region to promote Nrf2-MafK complex initiation of DNA activity. Also, JDP2 binds to ARE to promote expression of ARE-associated genes (Chiou et al., 2013; Tanigawa et al., 2013). Therefore, JDP2 plays an important role in protecting cells against oxidative stress. Expression of NQO1 and other antioxidant stress proteins also rely on expression of the JDP2 protein. In this study, JDP2 expression in NSC-34 cells transfected with TDP-43M337V plasmid was reduced, which might reduce expression of NQO1 and other antioxidant stress proteins, thereby impairing cellular responses to oxidative stress. Specific mechanisms underlying JDP effects on the Nrf2/ARE signaling pathway have not been elucidated and require further study.

In summary, NSC-34 cells transfected with pDEST30-EGFP-TDP-43-M337V showed impairment of Nrf2/ARE pathway. This resulted in reduced expression of the intracellular Nrf2 protein and the downstream phase II detoxifying enzyme, which then reduced antioxidant capacity and cell viability. Besides, results showed a reduction of MafK and JDP2 protein expression, which might cause a reduced expression of the downstream phase II detoxifying enzyme despite an aggregation of the nuclear Nrf2 protein. Since this study is still at an initial stage, a more in-depth study is needed to verify the findings.

\section{Conflicts of interest}

The authors declare no conflict of interest.

\section{ACKNOWLEDGMENTS}

Research supported in part by a grant from the Shandong Provincial Natural Science Foundation, China (\#ZR2010HM041) and a grant from the Shandong Provincial Post-Doctoral Innovation Foundation, China (\#201102004).

\section{REFERENCES}

Aronheim A, Zandi E, Hennemann H, Elledge SJ, et al. (1997). Isolation of an AP-1 repressor by a novel method for detecting protein-protein interactions. Mol. Cell. Biol. 17: 3094-3102 http://dx.doi.org/10.1128/MCB.17.6.3094.

Ayala YM, Zago P, D'Ambrogio A, Xu YF, et al. (2008). Structural determinants of the cellular localization and shuttling of TDP-43. J. Cell Sci. 121: 3778-3785 http://dx.doi.org/10.1242/jcs.038950.

Bozzo F, Mirra A and Carrì MT (2017). Oxidative stress and mitochondrial damage in the pathogenesis of ALS: New

Genetics and Molecular Research 16 (2): gmr16029638 
perspectives. Neurosci. Lett. 636: 3-8 http://dx.doi.org/10.1016/j.neulet.2016.04.065.

Brown JA, Min J, Staropoli JF, Collin E, et al. (2012). SOD1, ANG, TARDBP and FUS mutations in amyotrophic lateral sclerosis: a United States clinical testing lab experience. Amyotroph. Lateral Scler. 13: 217-222 http://dx.doi.org/10 $.3109 / 17482968.2011 .643899$.

Buendia I, Michalska P, Navarro E, Gameiro I, et al. (2016). Nrf2-ARE pathway: An emerging target against oxidative stress and neuroinflammation in neurodegenerative diseases. Pharmacol. Ther. 157: 84-104 http://dx.doi.org/10.1016/j. pharmthera.2015.11.003.

Chiou SS, Wang SS, Wu DC, Lin YC, et al. (2013). Control of Oxidative Stress and Generation of Induced Pluripotent Stem Cell-like Cells by Jun Dimerization Protein 2. Cancers (Basel) 5: 959-984 http://dx.doi.org/10.3390/cancers5030959.

Dhakshinamoorthy S and Jaiswal AK (2000). Small maf (MafG and MafK) proteins negatively regulate antioxidant response element-mediated expression and antioxidant induction of the $\mathrm{NAD}(\mathrm{P}) \mathrm{H}:$ Quinone oxidoreductase 1 gene. $J$. Biol. Chem. 275: 40134-40141 http://dx.doi.org/10.1074/jbc.M003531200.

Duan W, Li X, Shi J, Guo Y, et al. (2010). Mutant TAR DNA-binding protein-43 induces oxidative injury in motor neuronlike cell. Neuroscience 169: 1621-1629 http://dx.doi.org/10.1016/j.neuroscience.2010.06.018.

Jin C, Ugai H, Song J, Murata T, et al. (2001). Identification of mouse Jun dimerization protein 2 as a novel repressor of ATF-2. FEBS Lett. 489: 34-41 http://dx.doi.org/10.1016/S0014-5793(00)02387-5.

Jin C, Li H, Murata T, Sun K, et al. (2002). JDP2, a repressor of AP-1, recruits a histone deacetylase 3 complex to inhibit the retinoic acid-induced differentiation of F9 cells. Mol. Cell. Biol. 22: 4815-4826 http://dx.doi.org/10.1128/ MCB.22.13.4815-4826.2002.

Jin C, Kato K, Chimura T, Yamasaki T, et al. (2006). Regulation of histone acetylation and nucleosome assembly by transcription factor JDP2. Nat. Struct. Mol. Biol. 13: 331-338 http://dx.doi.org/10.1038/nsmb1063.

Kataoka K, Noda M and Nishizawa M (1994). Maf nuclear oncoprotein recognizes sequences related to an AP-1 site and forms heterodimers with both Fos and Jun. Mol. Cell. Biol. 14: 700-712 http://dx.doi.org/10.1128/MCB.14.1.700.

Katsuoka F and Yamamoto M (2016). Small Maf proteins (MafF, MafG, MafK): History, structure and function. Gene 586: 197-205 http://dx.doi.org/10.1016/j.gene.2016.03.058.

Katsuoka F, Motohashi H, Ishii T, Aburatani H, et al. (2005). Genetic evidence that small maf proteins are essential for the activation of antioxidant response element-dependent genes. Mol. Cell. Biol. 25: 8044-8051 http://dx.doi. org/10.1128/MCB.25.18.8044-8051.2005.

Katz S, Heinrich R and Aronheim A (2001). The AP-1 repressor, JDP2, is a bona fide substrate for the c-Jun N-terminal kinase. FEBS Lett. 506: 196-200 http://dx.doi.org/10.1016/S0014-5793(01)02907-6.

Kimura M, Yamamoto T, Zhang J, Itoh K, et al. (2007). Molecular basis distinguishing the DNA binding profile of Nrf2Maf heterodimer from that of Maf homodimer. J. Biol. Chem. 282: 33681-33690 http://dx.doi.org/10.1074/jbc. $\underline{\mathrm{M} 706863200 .}$.

Lagier-Tourenne C and Cleveland DW (2009). Rethinking ALS: the FUS about TDP-43. Cell 136: 1001-1004 http:// dx.doi.org/10.1016/j.cell.2009.03.006.

Li W and Kong AN (2009). Molecular mechanisms of Nrf2-mediated antioxidant response. Mol. Carcinog. 48: 91-104 http://dx.doi.org/10.1002/mc.20465.

Maekawa S, Leigh PN, King A, Jones E, et al. (2009). TDP-43 is consistently co-localized with ubiquitinated inclusions in sporadic and Guam amyotrophic lateral sclerosis but not in familial amyotrophic lateral sclerosis with and without SOD1 mutations. Neuropathology 29: 672-683 http://dx.doi.org/10.1111/j.1440-1789.2009.01029.x.

Mathis S, Couratier P, Julian A, Vallat JM, et al. (2017). Management and therapeutic perspectives in amyotrophic lateral sclerosis. Expert Rev. Neurother. 17: 263-276 http://dx.doi.org/10.1080/14737175.2016.1227705.

Neumann M, Sampathu DM, Kwong LK, Truax AC, et al. (2006). Ubiquitinated TDP-43 in frontotemporal lobar degeneration and amyotrophic lateral sclerosis. Science 314: 130-133 http://dx.doi.org/10.1126/science.1134108.

Rasmussen MH, Wang B, Wabl M, Nielsen AL, et al. (2009). Activation of alternative Jdp2 promoters and functional protein isoforms in T-cell lymphomas by retroviral insertion mutagenesis. Nucleic Acids Res. 37: 4657-4671 http:// dx.doi.org/10.1093/nar/gkp469.

Romano V, Quadri Z, Baralle FE and Buratti E (2015). The structural integrity of TDP-43 N-terminus is required for efficient aggregate entrapment and consequent loss of protein function. Prion 9: 1-9 http://dx.doi.org/10.1080/1933 $\underline{6896.2015 .1011885}$.

Scotter EL, Chen HJ and Shaw CE (2015). TDP-43 Proteinopathy and ALS: Insights into Disease Mechanisms and Therapeutic Targets. Neurotherapeutics 12: 352-363 http://dx.doi.org/10.1007/s13311-015-0338-x.

Tafuri F, Ronchi D, Magri F, Comi GP, et al. (2015). SOD1 misplacing and mitochondrial dysfunction in amyotrophic lateral sclerosis pathogenesis. Front. Cell. Neurosci. 9: 336 http://dx.doi.org/10.3389/fncel.2015.00336.

Tan CF, Eguchi H, Tagawa A, Onodera O, et al. (2007). TDP-43 immunoreactivity in neuronal inclusions in familial

Genetics and Molecular Research 16 (2): gmr16029638 
amyotrophic lateral sclerosis with or without SOD1 gene mutation. Acta Neuropathol. 113: 535-542 http://dx.doi. org/10.1007/s00401-007-0206-9.

Tanigawa S, Lee CH, Lin CS, Ku CC, et al. (2013). Jun dimerization protein 2 is a critical component of the Nrf2/ MafK complex regulating the response to ROS homeostasis. Cell Death Dis. 4: e921 http://dx.doi.org/10.1038/ cddis.2013.448.

Tateishi T, Hokonohara T, Yamasaki R, Miura S, et al. (2010). Multiple system degeneration with basophilic inclusions in Japanese ALS patients with FUS mutation. Acta Neuropathol. 119: 355-364 http://dx.doi.org/10.1007/s00401-0090621-1.

Taylor JP, Brown RH, Jr. and Cleveland DW (2016). Decoding ALS: from genes to mechanism. Nature 539: 197-206 http://dx.doi.org/10.1038/nature20413.

Tsai MH, Wuputra K, Lin YC, Lin CS, et al. (2016). Multiple functions of the histone chaperone Jun dimerization protein 2. Gene 590: 193-200 http://dx.doi.org/10.1016/j.gene.2016.03.048.

Wakabayashi N, Dinkova-Kostova AT, Holtzclaw WD, Kang MI, et al. (2004). Protection against electrophile and oxidant stress by induction of the phase 2 response: fate of cysteines of the Keap1 sensor modified by inducers. Proc. Natl. Acad. Sci. USA 101: 2040-2045 http://dx.doi.org/10.1073/pnas.0307301101.

Wang F, Lu Y, Qi F, Su Q, et al. (2014). Effect of the human SOD1-G93A gene on the Nrf2/ARE signaling pathway in NSC-34 cells. Mol. Med. Rep. 9: 2453-2458 10.3892/mmr.2014.2087.

Weidenfeld-Baranboim K, Hasin T, Darlyuk I, Heinrich R, et al. (2009). The ubiquitously expressed bZIP inhibitor, JDP2, suppresses the transcription of its homologue immediate early gene counterpart, ATF3. Nucleic Acids Res. 37: 21942203 http://dx.doi.org/10.1093/nar/gkp083.

Winton MJ, Igaz LM, Wong MM, Kwong LK, et al. (2008). Disturbance of nuclear and cytoplasmic TAR DNA-binding protein (TDP-43) induces disease-like redistribution, sequestration, and aggregate formation. J. Biol. Chem. 283: 13302-13309 http://dx.doi.org/10.1074/jbc.M800342200.

Yamazaki H, Tanji K, Wakabayashi K, Matsuura S, et al. (2015). Role of the Keap1/Nrf2 pathway in neurodegenerative diseases. Pathol. Int. 65: 210-219 http://dx.doi.org/10.1111/pin.12261.

Zarei S, Carr K, Reiley L, Diaz K, et al. (2015). A comprehensive review of amyotrophic lateral sclerosis. Surg. Neurol. Int. 6: 171. http://dx.doi.org/10.4103/2152-7806.169561

Genetics and Molecular Research 16 (2): gmr16029638 